\title{
DESAFÍOS DE TERAPIA OCUPACIONAL EN EL MANEJO DE SOBREPESO Y OBESIDAD
}
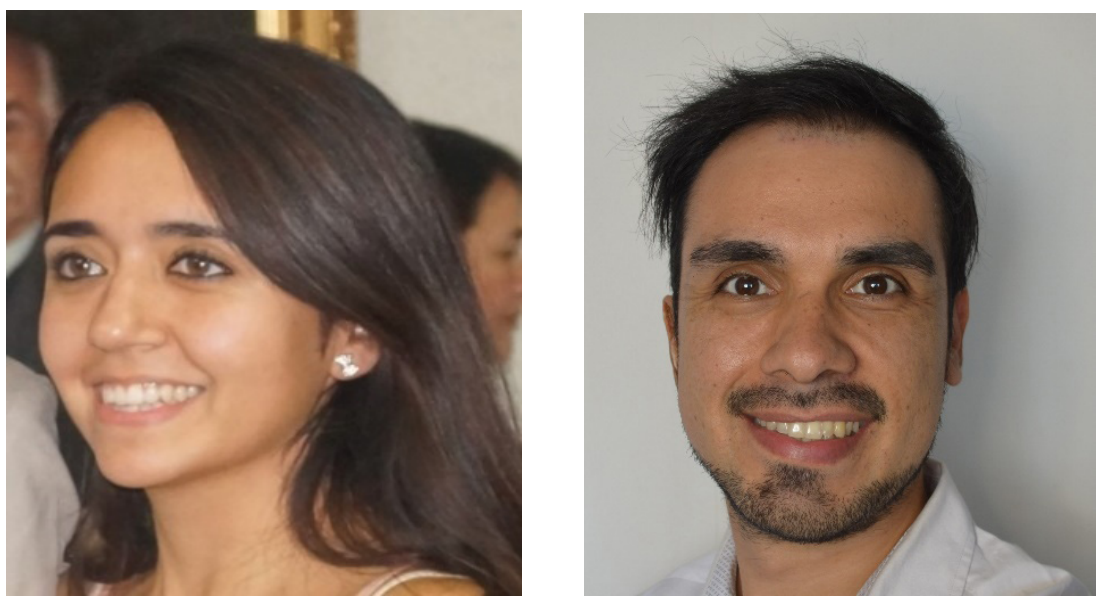

$\mathrm{E}$ n los últimos treinta años, la tasa de obesidad y sobrepeso han aumentado considerablemente a nivel mundial, por lo cual se ha convertido en un grave problema de Salud Pública, esto se debe a consecuencia - principalmente- de los estilos de vida y los hábitos alimenticios. Esto, nos obliga a establecer desafíos en la investigación y establecimiento de planes efectivos y eficientes para el manejo de esta condición. En la última Encuesta Nacional de Salud del año 2017 (Margozzini, Passi, 2018), el 74,2\% de la población chilena mayores de 15 años vive con sobrepeso u obesidad, situando a Chile, en el segundo lugar de los países de la OCDE (Organización para la Cooperación y el Desarrollo Económicos, 2020). Esto no es un dato menor, ya que esta situación se ha visto adicionalmente afectada en el contexto de la pandemia y el manejo CoviD-19, ya que las personas que viven con obesidad tienen un mayor riesgo de tener una peor evolución y mayores tasas de hospitalización. Incluso, un alto porcentaje de estos pacientes en las Unidades de Cuidados Intensivos tienen un mayor riesgo de mortalidad (Dana, Bannay, Bourst, et al., 2021).

Por otra parte, hemos visto que Chile ha desarrollado políticas públicas y programas relacionados al manejo de esta condición, los cuales se han enmarcan en los principalmente en los objetivos sanitarios de la década 2011-2020 (Ministerio de Salud, s,f), en donde se establece dentro de esta temática, por ejemplo, lograr resultados de mantención o disminución de la obesidad infantil en menores de 6 años en el sector público, sin embargo se ha observado un aumento del 9,8\% al 12,1\% en el periodo 2010-2019 (Ministerio de Salud, $\mathrm{s}, \mathrm{f}$.), lo que se define como una "meta en retroceso" dentro de los objetivos sanitarios. Como estrategias sanitarias, el estado chileno ha desarrollado e implementado programas asociados a estos objetivos, como la Ley $\mathrm{N}^{\circ} 20.670$ o también conocido como el "Sistema Elige Vivir Sano" enfocado en la promoción de la actividad física, alimentación, nutrición 
saludable y salud bucal; Ley $\mathrm{N}^{0} 20.606$, en el año 2012, la cual aborda la composición nutricional de los alimentos y su publicidad; y finalmente Ley $\mathrm{N}^{\circ} 20.379$ o el "Sistema Chile Crece Contigo", enfocado en la promoción de la actividad física, alimentación, nutrición saludable y salud bucal (Ministerio de Salud, 2013).

Cabe señalar, que existen diversos factores que inciden sobre los estilos de vida y los hábitos alimenticios, uno de ellos radica en que la obesidad no se distribuye uniformemente entre los grupos de población, sino que se definen en función de sus características demográficas y sociales, ${ }^{6}$ convirtiéndose no solo en un problema de salud, sino que también cultural, económico, medioambiental, político y educacional. En este último ámbito, se conoce la distribución del estado nutricional, según los años de estudios cursados, en donde el 43,2 \% son personas obesas en el grupo de menos de 8 años de escolaridad en comparación al $27 \%$ de las personas con 12 o más años de estudios. Sumado a esto, existe una disparidad en las tasas en relación a los patrones nutricionales y nivel de actividad física, tanto en el trabajo como en el tiempo libre, haciendo un llamado a la acción urgente para corregir los desequilibrios y aliviar la carga que viven las poblaciones más vulnerables.

Bajo este escenario entonces, ¿Cómo debiéramos enfrentar esta problemática desde nuestra línea disciplinar?

Variada es la evidencia y consensos que han planteado la necesidad de un tratamiento integral para el manejo de la obesidad y el sobrepeso, desde esta perspectiva, este fenómeno entendido como un proceso multifactorial, debe, por consecuencia, desarrollarse entre equipos y dispositivos multidisciplinares.

Específicamente desde el rol disciplinar y los alcances concretos a abordar desde la Terapia Ocupacional, se propone en primer lugar, realizar investigaciones que permitan identificar problemáticas situadas a la población chilena, revisiones bibliográficas o de literatura sobre las estrategias y programas actuales que abordan esta temática tanto en el ámbito de la infancia como adultez, enfocándose en conjunto a las comorbilidades asociadas y calidad de vida.

En segundo lugar, conocer los contextos específicos (cultural, social, físico y personal) que favorecen la ganancia de peso y su eventual repercusión en hábitos o estilos de vida no saludables.

Finalmente, y como eje principal del proceso de intervención, se encuentra la educación y modificación de hábitos y rutinas enmarcadas dentro de las diversas áreas de la ocupación; como lo son principalmente descanso y sueño, ocio y tiempo libre, educación y trabajo, desarrollando estrategias de resignificación. 


\section{ReFERENCIAS BibLIOGRÁFICAS}

1. Dana, R., Bannay, A., Bourst, P., Ziegler, C., Losser, M. R., Gibot, S., Levy, B., Audibert, G., \& Ziegler, O. (2021). Obesity and mortality in critically ill COVID-19 patients with respiratory failure. International journal of obesity. 45(9), 2028-2037. https://doi.org/10.1038/s41366-021-00872-9

2. Ley No20.379. Diario Oficial de la República de Chile, Santiago, Chile, 01 de septiembre de 2009.

3. Ley No20.606. Diario Oficial de la República de Chile, Santiago, Chile, 06 de junio de 2012.

4. Ley No20.670. Diario Oficial de la República de Chile, Santiago, Chile, 14 de mayo de 2013.

5. Margozzini P, Passi Á. (2018) Encuesta Nacional de Salud, ENS 2016-2017: un aporte a la planificación sanitaria y políticas públicas en Chile. ARS MEDICA. Rev Cienc Médicas.43(1):30-34. doi:10.11565/arsmed. v43i1.1354

6. Ministerio de Salud. (2013). Programa nacional de salud de la infancia con enfoque integral. Santiago, Chile. Recuperado de: https://diprece.minsal.cl/wrdprss_minsal/wp-content/uploads/2015/10/2013_ Programa-Nacional-de-Salud-de-la-infancia-con-enfoque-integral.pdf

7. Ministerio de Salud (s,f) Estrategia Nacional de Salud / Metas 2011-2020. http://www.bibliotecaminsal. cl/estrategia-nacional-de-salud-metas-2011-2020/

8. Ministerio de Salud (s,f) Resultado Indicadores de Salud - SIMPO. http://simpo.minsal.cl/monitoreo_indicadores/dashboard

9. Organización para la Cooperación y el Desarrollo Económicos. (2010). Obesity and the Economics of Prevention: Fit Not Fat. https://www.oecd-ilibrary.org/social-issues-migration-health/obesity-and-the-economics-of-prevention_9789264084865-en

10. Organización para la Cooperación y el Desarrollo Económicos. (2020). Discover the OECD: Together we create better policies for better lifes. http://www.oecd.org/general/Key-information-about-theOECD.pdf

Sebastián Gallegos B.

Profesor Asistente Departamento Terapia Ocupacional Universidad de Chile Terapeuta Ocupacional Hospital Clínico Universidad de Chile Terapeuta Ocupacional, Licenciado en Ciencias de la Ocupación, Magister Neurociencias

Rosa Ortiz E.

Terapeuta Ocupacional Departamento Terapia Ocupacional Universidad de Chile Terapeuta Ocupacional Hospital Clínico Universidad de Chile Terapeuta Ocupacional, Licenciada en Ciencias de la Ocupación, Magister en Ocupación y Terapia Ocupacional 